\title{
Focused Ion Beam (FIB) Microscopy and Technology
}

\author{
P. E. Russell and F. A. Stevie \\ Analytical Instrumentation Facility, Room 318 EGRC, Campus Box 7531, North \\ Carolina State University, Raleigh, NC 27695-7531
}

The course discusses the concepts and practical aspects of using focused ion beams (FIB) as a basis for microscopy and micromachining. The development of the high brightness gallium liquid metal ion source (LMIS) and associated ion optics has resulted in FIB microscope/workstations playing several major roles in microscopy and microanalysis laboratories. Figure 1 shows a LMIS with tungsten needle wetted with gallium from the reservoir.

FIB optics with $5 \mathrm{~nm}$ resolution performance is described and compared with a typical scanning electron microscope (SEM). The unique features of FIB due to the sputtering capabilities of the Ga beam are described. Contrast mechanisms when imaging with emitted electrons in a scanning ion beam system are presented with examples. FIG. 2 shows a FIB secondary electron image of a cross section ion milled to expose semiconductor metal layers. The metal grain structure can be easily observed. The interaction of ions with matter is presented to the extent required to understand the sputtering process and beam induced chemistry. Ion beam induced chemistry techniques are described which allow, with different chemistries, both material deposition with high spatial resolution and enhanced machining with increased material etch rates and/or selectivities. FIG. 3 shows an example of enhanced etch using water vapor to selectively remove polyimide to expose metal lines.

The use of FIB as a micromachining system for numerous applications is described. These applications include cross section of bulk samples and in-situ imaging, TEM sample preparation, and fabrication of probes for scanned probe microscopy (STM and AFM), see FIG. 4. Examples are also given for site specific TEM sample preparation including in situ and ex situ lift out methods that allow for low damage atomic resolution specimen preparation with rapid turn around time. FIG. 5 is a TEM micrograph from a FIB prepared specimen showing $\mathrm{Cu}$ metallization, barrier layers and vias. The course includes a description of the use of FIB to repair devices and provide circuit modifications. Figure 6 shows an example of an FIB cut and FIB induceddeposition to provide a re-routing of the line to another connection.

The course concludes with a brief discussion of current FIB instrumentation including systems with FE-SEM columns incorporated into a dual beam configuration. 


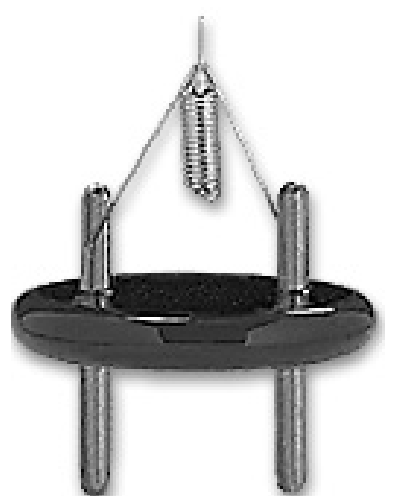

FIG. 1 LMIS

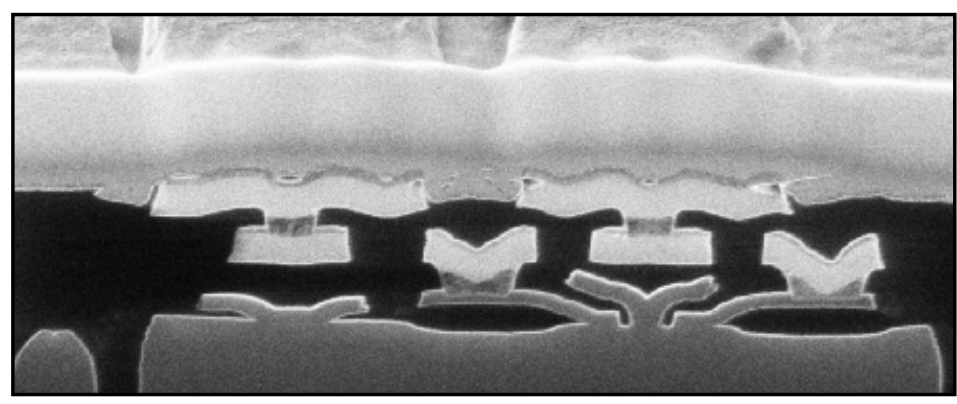

FIG. 2 FIB SEM image of cross section of semiconductor structure
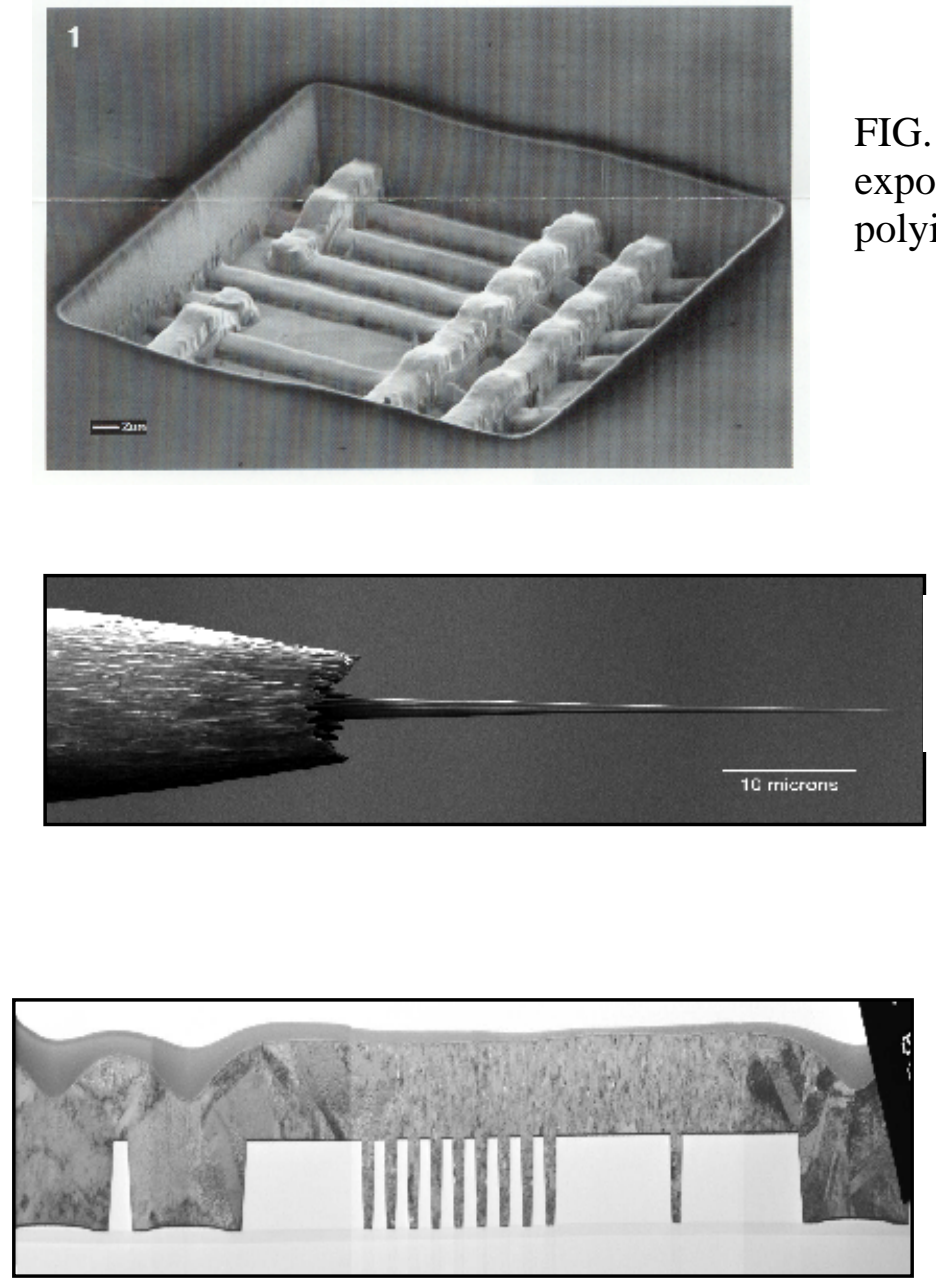

FIG. 3 Water enhanced etch to expose metal lines under polyimide
FIG. 4 FIB fabricated

AFM probe
FIG. 5 TEM micrograph of FIB prepared specimen showing $\mathrm{Cu}$ metallization

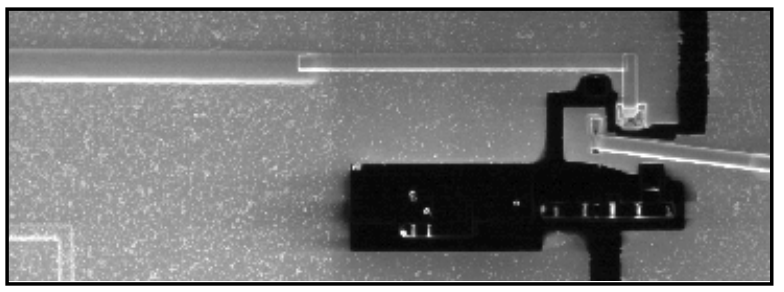

FIG. 6 FIB rerouting of semiconductor connecting line 